\title{
Psychological status of health care workers during the outbreak of Coronavirus disease in China: a cross-sectional study
}

\section{Xiangrui Song}

Naval Medical University https://orcid.org/0000-0002-2817-7903

\section{Chunyan Ni}

The Affiliate Suzhou Science\&Technoligy towen Hospital of Nanjing Medical University

\section{Wenpeng Cai}

Naval Medical University

\section{Tianya Hou}

Naval medical University

\section{Bin Lian}

The Affiliated Suzhou Science\&Technology Town Hospital of Nanjing Medical University

\section{Aibin Chen}

Naval Medical University

\section{Qianlan Yin}

Naval Medical University

Guanghui Deng ( $\sim$ guanghuideng@163.com )

Naval Medical University

Huifen Li ( $\square$ 1927280851@qq.com )

The Affiliated Suzhou Science \& Technology Town Hospital of Nanjing Medical University

\section{Research article}

Keywords: Health care workers, Psychological status, Mental health, Resilience, Social support

Posted Date: March 23rd, 2020

DOl: https://doi.org/10.21203/rs.3.rs-18641/v1

License: (c) (i) This work is licensed under a Creative Commons Attribution 4.0 International License. Read Full License 


\section{Abstract}

Background: Following the outbreak of Coronavirus disease (COVID-19) in Wuhan, China, thousands of health care workers (HCWs) joined in the battle to prevent epidemic. The purposes of this study were to assess the psychological status of health care workers fighting against COVID-19 and compared their status with non-health care workers.

Methods: 1521 participants were invited to complete a cross-sectional survey which consisted of a demographic questionnaire, the symptom checklist-90 (SCL-90), the Connor-Davidson Resilience scale (CD-RISC) and Chinese-Version Social support revalued scale (SSRS). SCL-90 was used as a measure of psychological status, CD-RISC was used as a measure of resilience and SSRS was used as a measure of social support. All analyses were completed by SPSS 21.0 and two-tailed with significance defined as $p$ $<0.05$.

Results: HCWs showed higher level of obsessive-compulsive symptoms ( $p=0.002)$, depression ( $p$ $=0.011)$, anxiety $(p=0.037)$ and had lower level of subjective support $(p<0.001)$ as well as strength $(p$

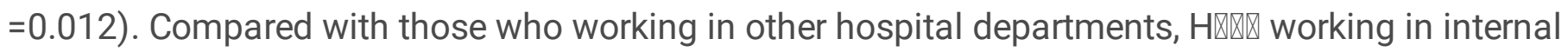
medicine department had high level of obsessive-compulsive, anxiety and interpersonal sensitivity, they also had low level of social support and resilience.

Conclusions: HCWs were vulnerable to mental disorders, and health organizations and government should initiate psychological assistant program to keep them immune to mental disorders. HCWs who working in internal medicine department (IMD) were supposed to rebuild their resilience, social support systems, confidence and job satisfaction under the guidance of psychologists.

\section{Background}

Following the development of the COVID-19 in Wuhan, more and more people were infected by this disease. There were 78497 confirmed patients affected by this novel coronavirus and 2744 patients died by February 26, 2020 in China [1]. Moreover, this Novel coronavirus had spread to many other countries including Japan, South Korea, and Italy. it had been classified as a major public health event by World Health Organization (WHO). Millions of volunteers mobilized by health organizations and government, including HCWs and social workers who were responsible for public education, quarantine or other work in the battle of preventing epidemic. Previous studies indicated that HCWs presented more absenteeism and reduced their willingness of face to face diagnosis and treatment to patients during the outbreak of Sever Acute Respiratory Syndrome (SARS) [2]. Furthermore, studies showed that HCWs suffered depression, anxiety and sleep disorders during the outbreak of SARS [3] Stress can be brought by major life events and impact psychological health, especially the major public events which result in loss of property and lives. Epidemics, like SARS and influenza, as one of the major public health events, could be one of most significant stressors for HCWs working on the frontline and highly exposed to the pathogen. 
Generally, resilience was defined as the ability to cope with stressors and difficulties, maintain psychological functioning and prevent the damages form stressors and hardness[5]. High resilience can help people maintain the psychological well-being[6] and recover from setbacks quickly [7-9]. Meanwhile, Social support was considered as a protective factor for person experiencing crises and challenges $[5,9-$ $11]$, it reflected the relationships between people and their family members, friends, colleagues as well as organizations which they worked. Previous studies found that people had high level of social support also had high level of mental health and it can help developing resilience[12].

To investigate the psychological health status of HCWs and non-HCWs who engaged in public education and quarantine as well as instruction in this epidemic prevention, we designed this study to evaluate their mental health and resilience as well as social support. Furthermore, we wondered whether this epidemic would affect HCWs' psychological status working in different hospital departments during the outbreak of epidemic. According to previous studies about SARS and other epidemics, we hypothesized that HCWs suffering more psychological stress and would be more likely to be a bad mental status.

\section{Method}

\section{Participants}

1521 participants were invited to take part in this study. Working in Anhui province of China where 989 patients affected and 6 died by February 26, 2020. Subjects of samples consisted of HCWs from different hospital departments and non-HCWs who engaged in public education, quarantine or other work.

\section{Measures}

\section{The symptom checklist-90}

Compiled by Derogatis LR in 1973[13], The symptom checklist-90 (SCL-90) consists of 90 items and includes a wide range of psychiatric symptomatology content, from sensory, emotion, thinking, consciousness, behavior to living habits, interpersonal relations, diet and sleep, etc.. From no such problem to the frequency and intensity of the symptom is very serious, each of item adopts a 5-level (1-5) rating system. If one subscale score is higher than 2 , the number of positive items is higher than 43 , or the total score is higher than 160, it suggests psychological abnormality. Previous study indicated that it has high reliability and validity[14].

\section{The Connor-Davidson Resilience scale (CD-RISC)}

CD-RISC was compiled by Kathryn M. Connor and Jonathan R.T. Davidson in 2003[15]. Consists of 25 items, CD-RISC is one of most significant measures to invest resilience. Chinese version CD-RISC which in accordance to behavior habits of Chinese people was revised by Xiao et al. in 2007,Each item rate on five points (0-4) and the options provide for subjects list as follows: 0 , not true at all; 1 , rarely true; 2 , sometimes true; 3 , often true; 4 , nearly true all the time. The scores range from 0 to 100 , higher sores reflect participants have greater resilience and lower scores reflect lesser resilience[15]. Three subscales 
of Chinese version CD-RISC named as tenacity, strength and optimism. Previous studies showed that it exhibited strong reliability and validity as well as the predictive ability [15-18].

\section{Chinese Version Social support revalued scale (SSRS)}

Chinese version of social support revalued scale was compiled by Suiyuan Xiao in 1999.it comprises of 10 items and three subscales, namely, subjective support, objective support and social support utilization. By calculating the sum of item scores on different subscales, the actual objective support, subjective experience, and individual utilization of social support are measured. Previous study indicated that it has high reliability and validity[19].

\section{Statistical analysis}

All data analyses were carried out by the SPSS 21.0 (SPSS Inc, Chicago, IL) for windows.

Frequency, percentage means and standard deviations were calculated for descriptive data. Independent sample T-test was conducted to compare the outcomes between HCWs and non-HCWs. One-way ANOVA, test of Homogeneity of variances and post hoc test were conducted to investigate the differences of HCWs working in different hospital departments. $\mathrm{P} \otimes 0.05$ was considered statistically significant.

\section{Results}

\section{Descriptive data}

A total of 1521 subjects completed this survey. The descriptive statistics of demographic data were shown in Table1. Samples consisted of $1198 \mathrm{HCW}$ and 323 non-HCWs who engaged in fighting this epidemic; There were 372 males and 1149 females took part in this study; 1123 participants were married, 380 participants were unmarried, 18 participants were at other situations; 132 participants worked in surgery department (SD),144 participants worked in internal medicine department (IMD), 67 participants worked in obstetrics and gynecology department (OGD), 41 participants worked in emergence room (ER), 32 participants worked in intensive care unit (ICU), 69 participants worked in outpatient department (OPD), 27 participants worked in pediatrics department (PD), 1participant worked in administration department, 7 participants worked in anesthesiology department (AD) and 975 participants didn't report.

\section{Group differences between HCWs and non-HCWs}

As shown in Table2, the outcomes of three scales between HCWS and non-HCWs were compared, there were significant differences on obsessive-compulsive $(p=0.002)$, depression $(p=0.011)$, anxiety $(p=0.037)$, subjective support $(p<0.001)$, strength $(p=0.012)$ and no significant difference on other subscales. It indicated HCWs suffered more anxiety, depression, obsessive- compulsive, lower subjective support and strength. 


\section{Group difference between different hospital department of HCWs}

As shown in Table3, we compared the outcomes of HCWs working in different hospital departments on all subscales with one-way ANOVA. It indicated that there were significant differences in subscales of objective support ( $p=0.039)$ and subjective support $(p=0.007)$ as well as total score of social support ( $p$ $=0.034)$ and no significant differences on other subscales.

We further conducted post hoc test to explorer the differences among HCWs working in different hospital departments and we found that there were significant differences among HCWs on some subscales. HCWs working in IDP had higher level of obsessive-compulsive symptoms than those working in ICU ( $p$ $=0.040)$ and OPD $(p=0.001)$; HCWs working in OGD had higher level obsessive-compulsive symptoms than those who working in OPD $(p=0.034)$; HCWs working in IMD had higher level than those working in OPD on the subscales of obsessive compulsive $(p=0.033)$ and anxiety $(p=0.037)$ as well as interpersonal sensitivity $(p=0.033)$. There was no significant difference among HCWs on other subscales of SCL-90.

Post hoc test revealed that there were several significant differences in HCWs working in different hospital departments on the subscales of SSRS. HCWs working in AD had lower level of objective support than HCWs who working in SD $(p=0.018)$; HCWs working in OPD had higher level of subjective support than HCWs who working in IMD $(p<0.001), \operatorname{OGD}(p=0.016)$, ER $(p=0.014)$ and ICU $(p=0.002)$; HCWs working in SD had higher total score of SSRS than HCWs who working in IMD ( $p=0.001)$, OGD ( $p=$ $0.031)$ and $\operatorname{ER}(p=0.035)$.

Beside differences on subscales of SCL-90 and SSRS, HCWs working in different hospital departments also had differences on subscales of CD-RISC. HCWs working in IMD had lower level of tenacity than those who working in SD $(p=0.007)$ and OPD $(p=0.008)$, but there was no significant difference among HCWs working in other departments on this subscale. Moreover, HCWs working in IMD had lower level of strength than those working in SD $(p=0.012), \operatorname{ICU}(p=0.046), \operatorname{OPD}(p=0.023)$ and $\operatorname{AD}(0.028)$, but there was no significant difference among HCWs working in other departments on this subscale. HCWs working in IMD had lower level of optimism than those who working in ICU $(p=0.042)$ and OPD $(p=$ 0.004), but there was no significant difference among HCWs working in other departments on this subscale. HCWs working in IMD had lower resilience than those who working in SD $(p=0.011)$, ICU ( $p=$ $0.046)$ and OPD $(p=0.007)$, but there was no significant difference among HCWs working in other departments on this subscale.

\section{Discussion}

In this study, we tested the psychological status and resilience as well as social support of volunteers who engaged in fighting this epidemic during the peak period, we compared the outcomes of HCWs and non-HCWs and then, we tested the differences among HCWs working in different hospital departments on all the subscales of SCL-90, SSRS and CD-RISC. Result showed that psychological status of HCWs were worse than non-HCWs on subscales of obsessive-compulsive, depression and anxiety. It indicated that 
HCWs suffered more stress, negative emotions and burnout than non-HCWs. The comparisons among HCWs working in different hospital departments showed that HCWs working in IMD had higher level of obsessive-compulsive, interpersonal sensitivity and anxiety on SCL-90 and lower level of subjective support, objective support on SSRS as well as lower level of tenacity, optimism and resilience on CD-RISC.

Different studies have documented that HCWs suffer more stress during the outbreak of SARS[20-23]. HCWs were backbone in the battle toward this epidemic, however, HCWs were at high risk of being affected. Studies showed that 20 percentage of patients affected SARS were HCWs during the outbreak of SARS in Hongkong[24], and there were more than $3000 \mathrm{HCWs}$ affected and 5 died by the date of February 10, 2020. Unfortunately, Wenliang Li, one of the first doctors who realized the outbreak of epidemic, died in February 10, 2020. HCWs treated different patients coming from everywhere without full information about whether they were affected during the peak of epidemic and crowd patients easily lead to cross affection. HCWs who engaged in face to face diagnose and treatments needed wear protective clothing, surgical masks, medical eye proctors and other necessary protective equipments those were uncomfortable for their performance. worsely, working long hours and tremendous work stress rendered physicians and psychologists tired out $[25,26]$. As we discussed above, worrying about being affected and enduring harsh working environments as well as enormous workload were the significant stressors for HCWs and account for the low level of mental health.

Patients affected mainly presented cough, fever and symptoms of pneumonia, which were classical characteristics of respiratory symptoms, internal medicine physicians and nurses could be the first and main HCWs connected with these patients with respiratory symptoms, so HCWs working in IMD suffered more stress than those working in other departments for they were at higher risk of being affected, which explained why they presented more symptoms of obsessive-compulsive, interpersonal sensitivity and anxiety in this investigate. However, the reasons result in their low levels of social support and resilience need further explore and we observed that diagnosis for patients coming to IMD were frequently ambiguous and internal medicine physicians must try to judge them by experience and knowledge at the first stage, they should have to keep conventional treatments until clear clinic diagnoses were developed, they may have to overturn their diagnosis during this procedure and their self-confidence would be impacted during long-term medical work. Moreover, working in IMD was relatively monotonous and lack of challenges, sense of achievements and job satisfactions were deficient. The impacts of work environment, work characteristic and job satisfactions to social support and resilience of HCWs working in IMD were study emphases in our further research. Previous studies showed that social support and resilience were protective factors of mental health[27-30], but this study showed that HCWs working in IMD presented low levels of social support and resilience which could partly explain their more symptoms of obsessive-compulsive, interpersonal sensitivity and anxiety[31, 32].

This study investigated the psychological status of volunteers battling in this epidemic at the peak period, we found that HCWs had worse mental health status than non-HCWs, especially HCWs working in IMD who suffered more than those in other hospital departments, reported less social support and lower 
resilience. However, we didn't collect data of volunteers before the outbreak, so there was a lack of controls for other potential variables impacting the psychological effect of this epidemic.

\section{Conclusion}

HCWs need particularly psychological assistance program to help them keeping mental health during the outbreak of major health events, especially internal medicine physicians, they need Comprehensive psychological help program to rebuild their resilience, social support systems, confidence and job satisfaction.

\section{Abbreviations}

COVID-19: Coronavirus disease HCWs: health care workers; SARS: Sever Acute Respiratory Syndrome; SCL-90: The Symptom of Checklist-90; SSRS: The Social Support revalued Scale; CD-RISC: The ConnorDavidson Resilience scale; SD: surgery department; IMD: internal medicine department; OGD: obstetrics and gynecology department; ER: emergency room; ICU: intensive care unit; OPD: out-patient department; PD: pediatrics department; AP: Anesthesiology department.

\section{Declarations}

\section{Ethics approval and consent to participant}

This study was approved by the ethics committees of Naval Medical University, Shanghai, China. Informed written consent was obtained before survey.

\section{Consent for publication}

Not applicable

\section{Availability of data and materials}

The datasets used and/or analyzed during the current study are available from the corresponding author on reasonable request.

\section{Competing interests}

The authors declare that they have no competing interests

\section{Funding}

This study was funded by a larger project of military $13^{\text {th }}$ five-years plan, project number: BWS16J012. The authors declare that the funding body had no role in the design of the study, collection, analysis, and interpretation of data nor in writing the manuscript. 
Acknowledgements

Not applicable.

Author details

Affiliations

${ }^{1}$ Faculity of Psychology, Naval Medical University (Second Military Medical University), Shanghai 200433, China. ${ }^{2}$ Nusing Department, The Affiliated Suzhou Science \& Technology Town Hospital of Nanjing Medical University, Suzhou 215153, China

\section{Authors' contributions}

Design of study: All authors. Analyses: All authors. Drafting manuscript: Xiangrui Song. Critical revisions: Xiangrui Song and Chunyan Ni. Final Approval: All authors.

Corresponding author

Correspondence to Huifen Li or Guanghui Deng.

\section{References}

1. Feb 27: Daily briefing on novel coronavirus cases in China [http://en.nhc.gov.cn/202002/27/c_76975.htm]

2. Aiello A, Khayeri MY, Raja S, Peladeau N, Romano D, Leszcz M, Maunder RG, Rose M, Adam MA, Pain $\mathrm{C}$ et al: Resilience training for hospital workers in anticipation of an influenza epidemic. $J$ Contin Educ Health Prof 2011, 31(1):15-20.

3. Chen R, Chou KR, Huang YJ, Wang TS, Liu SY, Ho LY: Effects of a SARS prevention programme in Taiwan on nursing staff's anxiety, depression and sleep quality: a longitudinal survey. Int J Nurs Stud 2006, 43(2):215-225.

4. Li MY, Yang YL, Liu L, Wang L: Effects of social support, hope and resilience on quality of life among Chinese bladder cancer patients: a cross-sectional study. Health Qual Life Outcomes 2016, 14:73.

5. Farber EW, Schwartz JA, Schaper PE, Moonen DJ, McDaniel JS: Resilience factors associated with adaptation to HIV disease. Psychosomatics 2000, 41(2):140-146.

6. Jalilianhasanpour R, Williams B, Gilman I, Burke MJ, Glass S, Fricchione GL, Keshavan MS, LaFrance WC, Jr., Perez DL: Resilience linked to personality dimensions, alexithymia and affective symptoms in motor functional neurological disorders. $J$ Psychosom Res 2018, 107:55-61.

7. Lu W, Wang Z, You X: Physiological responses to repeated stress in individuals with high and low trait resilience. Biol Psychol 2016, 120:46-52. 
8. Pietrzak RH, Johnson DC, Goldstein MB, Malley JC, Southwick SM: Psychological resilience and postdeployment social support protect against traumatic stress and depressive symptoms in soldiers returning from Operations Enduring Freedom and Iraqi Freedom. Depress Anxiety 2009, 26(8):745-751.

9. Dave Sells WHS, Melissa Wielandy, David Waldenz, Elizabeth Flanagan, Rebecca Miller, Larry Davidson: Cascading crises, resilience and social support within the onset and development of multiple chronic conditions. Chronic Illness 2009(5): 92-102.

10. Havermans BM, Boot CRL, Houtman ILD, Brouwers EPM, Anema JR, van der Beek AJ: The role of autonomy and social support in the relation between psychosocial safety climate and stress in health care workers. BMC Public Health 2017, 17(1):558.

11. Fuentes-Peláez N, Balsells MÀ, Fernández J, Vaquero E, Amorós P: The social support in kinship foster care: a way to enhance resilience. Child \& Family Social Work 2016, 21(4):581-590.

12. Derogatis LR, Lipman RS, Covi L: SCL-90: an outpatient psychiatric rating scale-preliminary report. 1973, 9(1):13-28.

13. M. M. Holi PRS, V. A. Aalberg: A Finnish validation study of the SCL-90. Acta Psychiatr Scand 1998, 97:42-46.

14. Connor KM, Davidson JR: Development of a new resilience scale: the Connor-Davidson Resilience Scale (CD-RISC). Depress Anxiety 2003, 18(2):76-82.

15. Bezdjian S, Schneider KG, Burchett D, Baker MT, Garb HN: Resilience in the United States Air Force: Psychometric properties of the Connor-Davidson Resilience Scale (CD-RISC). Psychol Assess 2017, 29(5):479-485.

16. Zhang XYJ: Factor analysis and Psychometric evaluation of the connor-davidson resilience scale (cd-risc) with chinese PeoPle. SOCIAL BEHAVIOR AND PERSONALITY 2007, 35(1):19-30.

17. Khoshouei MS: Psychometric Evaluation of the Connor-Davidson Resilience Scale (CD-RISC) Using Iranian Students. International Journal of Testing 2009, 9(1):60-66.

18. Yunyong L, Zhe W, Junting X, Yan Z, Xiaoxia A, Li Z, Yuan G, Chao J: Associations between recent gay-related stressful events, emotional distress, social support and unprotected anal intercourse behavior among Chinese men who have sex with men. Aust N Z J Psychiatry 2016, 50(7):659-666.

19. Tai W. Wong JKYY, Cecilia L.W. Chan, Rosalie S.Y. Kwong, Samuel M.Y. Ho, Chor C. Lau, Fei L. Lau, Chau H. Lit: The psychological impact of severe acute respiratory syndrome outbreak on healthcare workers in emergency departments and how they cope. European Journal of Emergency Medicine 2005, 12(1):13-18.

20. Tam CW, Pang EP, Lam LC, Chiu HF: Severe acute respiratory syndrome (SARS) in Hong Kong in 2003: stress and psychological impact among frontline healthcare workers. Psychol Med 2004, 34(7):1197-1204.

21. Wong JG, Cheung EP, Cheung V, Cheung C, Chan MT, Chua SE, McAlonan GM, Tsang KW, Ip MS: Psychological responses to the SARS outbreak in healthcare students in Hong Kong. Med Teach 2004, 26(7):657-659. 
22. Lu YC, Shu BC, Chang YY, Lung FW: The mental health of hospital workers dealing with severe acute respiratory syndrome. Psychother Psychosom 2006, 75(6):370-375.

23. Su T, Lien T, Yang C, Su Y, Wang J, Tsai S, Yin J: Prevalence of psychiatric morbidity and psychological adaptation of the nurses in a structured SARS caring unit during outbreak: $A$ prospective and periodic assessment study in Taiwan. Journal of Psychiatric Research 2007, 41(12):119-130.

24. Conway PM, Campanini P, Sartori S, Dotti R, Costa G: Main and interactive effects of shiftwork, age and work stress on health in an Italian sample of healthcare workers. Appl Ergon 2008, 39(5):630639.

25. JEFFREY V. JOHNSON AEMH: Job Strain, Work Place Social Support, and Cardiovascular Disease: A Cross-Sectional Study of a Random Sample of the Swedish Working Population. AJPH1988, 78(10):1336-1342.

26. Pengju WANG ZX, Hua YANG: Relationship of Mental Health, Social Support, and Coping Styles among Graduate Students: Evidence from Chinese Universities. Iran J Public Health 2018, 47(5):.689697.

27. Horton TV, Wallander JL: Hope and social support as resilience factors against psychological distress of mothers who care for children with chronic physical conditions. Rehabilitation Psychology 2001, 46(4):382-399.

28. Yu Y, Peng L, Chen L, Long L, He W, Li M, Wang T: Resilience and social support promote posttraumatic growth of women with infertility: the mediating role of positive coping. Psychiatry Res 2014, 215(2):401-405.

29. Bert N. Uchino JTC, Janice K. Kiecolt-Glaser: The Relationship Between Social Support and Physiological Processes: A Review With Emphasis on Underlying Mechanisms and Implications for Health. Psychological Bulletin 1996, 119( 3):488-531.

30. Plaisier I, de Bruijn JG, de Graaf R, ten Have M, Beekman AT, Penninx BW: The contribution of working conditions and social support to the onset of depressive and anxiety disorders among male and female employees. Soc Sci Med 2007, 64(2):401-410.

31. Schultz P, Roditti M, Gillette M: Resilience, Social Support, and Psychological Disturbance in Hispanic Women Residing in a Battered Women's Shelter on the U.S./Mexico Border. Hispanic Health Care International 2009, 7(4):224-230.

\section{Tables}

Table1. Descriptive statistics of subjects' demographic data 


\begin{tabular}{|c|c|c|c|}
\hline Variable & Category & Frequency & Percentage \\
\hline \multirow[t]{2}{*}{ Gender } & male & 372 & 24.5 \\
\hline & female & 1149 & 75.5 \\
\hline \multirow[t]{6}{*}{ Age } & $18 \sim 25$ & 208 & 13.7 \\
\hline & $26 \sim 30$ & 454 & 29.8 \\
\hline & $31 \sim 40$ & 583 & 38.3 \\
\hline & $41 \sim 50$ & 227 & 14.9 \\
\hline & $51 \sim 60$ & 41 & 2.7 \\
\hline & $>60$ & 8 & 0.5 \\
\hline \multirow[t]{3}{*}{ Marital status } & Married & 1123 & 73.8 \\
\hline & Unmarried & 380 & 25 \\
\hline & Other & 18 & 1.2 \\
\hline \multirow[t]{4}{*}{ Offspring } & 0 & 516 & 33.9 \\
\hline & 1 & 718 & 47.2 \\
\hline & 2 & 285 & 18.7 \\
\hline & 3 & 2 & 0.1 \\
\hline \multirow[t]{4}{*}{ Education } & High school or lower & 320 & 21 \\
\hline & Undergraduate & 1266 & 83.2 \\
\hline & postgraduate or higher & 225 & 14.7 \\
\hline & Other & 4 & 0.3 \\
\hline \multirow[t]{2}{*}{ Health care worker } & Yes & 1198 & 78.8 \\
\hline & No & 323 & 21.2 \\
\hline \multirow[t]{6}{*}{ Occupation } & Doctor & 211 & 13.9 \\
\hline & Nurse & 546 & 35.9 \\
\hline & Pharmacist & 45 & 3 \\
\hline & Pharmacist & 72 & 4.7 \\
\hline & Logistical personnel & 136 & 8.9 \\
\hline & Social worker & 511 & 33.6 \\
\hline \multirow[t]{8}{*}{ workplace } & Surgical department & 132 & 8.7 \\
\hline & Internal medicine department & 144 & 9.5 \\
\hline & Obstetrics and gynecology department & 67 & 8.7 \\
\hline & Emergency room & 41 & 9.5 \\
\hline & Intensive care unit & 32 & 4.4 \\
\hline & Out-patient department & 69 & 2.7 \\
\hline & Pediatrics department & 27 & 2.1 \\
\hline & Administrative department & 7 & 4.5 \\
\hline
\end{tabular}

Page $11 / 13$ 


\begin{tabular}{lcc}
\hline Anesthesiology department & 27 & 1.8 \\
\hline Don't report & 975 & 64.1 \\
\hline
\end{tabular}

Table2.Comparisons between HCWs and non-HCWs on all subscales

\begin{tabular}{|c|c|c|c|c|}
\hline Subscale & HCWs & Non-HCWs & $\mathrm{t}$ & $\mathrm{p}$ (2-tailed) \\
\hline Somatization & $1.14 \pm 0.31$ & $1.12 \pm 0.31$ & 0.93 & 0.352 \\
\hline Obsessive-compulsive & $1.41 \pm 0.46$ & $1.33 \pm 0.39$ & 3.08 & 0.002 \\
\hline Interpersonal sensitivity & $1.26 \pm 0.40$ & $1.22 \pm 0.36$ & 1.62 & 0.106 \\
\hline Depression & $1.25 \pm 0.40$ & $1.19 \pm 0.35$ & 2.54 & 0.011 \\
\hline Anxiety & $1.22 \pm 0.36$ & $1.18 \pm 0.32$ & 2.09 & 0.037 \\
\hline Hostility & $1.23 \pm 0.36$ & $1.19 \pm 0.36$ & 1.68 & 0.093 \\
\hline Phobic anxiety & $1.20 \pm 0.37$ & $1.17 \pm 0.35$ & 1.41 & 0.158 \\
\hline Paranoid ideation & $1.18 \pm 0.33$ & $1.16 \pm 0.32$ & 0.7 & 0.482 \\
\hline Psychoticism & $1.16 \pm 0.32$ & $1.14 \pm 0.31$ & 0.95 & 0.345 \\
\hline Others & $1.23 \pm 0.36$ & $1.22 \pm 0.39$ & 0.59 & 0.557 \\
\hline Total score of social support & $110.85 \pm 30.13$ & $107.44 \pm 27.42$ & 1.839 & 0.066 \\
\hline Objective support & $10.82 \pm 3.73$ & $10.62 \pm 4.17$ & 0.82 & 0.421 \\
\hline Subjective support & $24.59 \pm 4.97$ & $25.88 \pm 4.80$ & -4.16 & $<0.001$ \\
\hline Support utilization & $8.28 \pm 1.98$ & $8.41 \pm 2.15$ & -0.98 & 0.323 \\
\hline Tenacity & $34.53 \pm 8.20$ & $35.41 \pm 8.68$ & -1.68 & 0.093 \\
\hline Strength & $23.50 \pm 4.90$ & $24.28 \pm 5.03$ & -2.51 & 0.012 \\
\hline Optimism & $10.09 \pm 2.68$ & $10.05 \pm 2.83$ & 0.27 & 0.789 \\
\hline Resilience & $68.13 \pm 14.75$ & $69.73 \pm 15.29$ & -1.723 & 0.085 \\
\hline
\end{tabular}

Table3. Comparisons among HCWs working in different hospital departments in all subscales 


\begin{tabular}{|c|c|c|c|c|c|c|c|c|c|}
\hline & IMD & OGD & ER & ICU & OPD & $\mathrm{PD}$ & AP & $\mathrm{F}$ & $\mathrm{p}$ \\
\hline 25 & $1.14 \pm 0.31$ & $1.15 \pm 0.36$ & $1.10 \pm 0.19$ & $1.16 \pm 0.21$ & $1.12 \pm 0.28$ & $1.19 \pm 0.45$ & $1.17 \pm 0.33$ & 0.34 & 0.935 \\
\hline 12 & $1.46 \pm 0.45$ & $1.42 \pm 0.48$ & $1.30 \pm 0.34$ & $1.43 \pm 0.42$ & $1.26 \pm 0.38$ & $1.39 \pm 0.47$ & $1.42 \pm 0.49$ & 1.88 & 0.071 \\
\hline 37 & $1.28 \pm 0.40$ & $1.29 \pm 0.44$ & $1.17 \pm 0.27$ & $1.17 \pm 0.24$ & $1.16 \pm 0.33$ & $1.25 \pm 0.44$ & $1.31 \pm 0.42$ & 1.33 & 0.236 \\
\hline 10 & $1.27 \pm 0.44$ & $1.27 \pm 0.43$ & $1.20 \pm 0.24$ & $1.17 \pm 0.25$ & $1.18 \pm 0.34$ & $1.25 \pm 0.47$ & $1.22 \pm 0.34$ & 0.65 & 0.713 \\
\hline 36 & $1.25 \pm 0.36$ & $1.22 \pm 0.39$ & $1.16 \pm 0.23$ & $1.20 \pm 0.27$ & $1.14 \pm 0.29$ & $1.24 \pm 0.44$ & $1.26 \pm 0.41$ & 0.88 & 0.524 \\
\hline 31 & $1.23 \pm 0.34$ & $1.26 \pm 0.48$ & $1.17 \pm 0.24$ & $1.19 \pm 0.24$ & $1.15 \pm 0.31$ & $1.30 \pm 0.49$ & $1.28 \pm 0.46$ & 1.02 & 0.413 \\
\hline 39 & $1.20 \pm 0.33$ & $1.20 \pm 0.42$ & $1.11 \pm 0.21$ & $1.12 \pm 0.22$ & $1.15 \pm 0.36$ & $1.24 \pm 0.50$ & $1.23 \pm 0.40$ & 0.72 & 0.658 \\
\hline 27 & $1.18 \pm 0.35$ & $1.20 \pm 0.40$ & $1.09 \pm 0.16$ & $1.16 \pm 0.24$ & $1.09 \pm 0.28$ & $1.19 \pm 0.45$ & $1.20 \pm 0.35$ & 1.03 & 0.408 \\
\hline 30 & $1.19 \pm 0.35$ & $1.17 \pm 0.38$ & $1.09 \pm 0.14$ & $1.10 \pm 0.17$ & $1.09 \pm 0.27$ & $1.16 \pm 0.44$ & $1.20 \pm 0.34$ & 1.04 & 0.400 \\
\hline 37 & $1.25 \pm 0.38$ & $1.26 \pm 0.42$ & $1.19 \pm 0.25$ & $1.18 \pm 0.24$ & $1.17 \pm 0.29$ & $1.22 \pm 0.45$ & $1.30 \pm 0.40$ & 0.74 & 0.640 \\
\hline 28.81 & $112.58 \pm 30.33$ & $112.10 \pm 34.90$ & $104.83 \pm 15.75$ & $108.13 \pm 19.07$ & $104.07 \pm 25.91$ & $112.11 \pm 39.05$ & $113.30 \pm 32.45$ & 0.90 & 0.504 \\
\hline .37 & $10.63 \pm 3.70$ & $10.75 \pm 3.93$ & $10.39 \pm 4.25$ & $11.88 \pm 4.64$ & $10.87 \pm 3.76$ & $10.85 \pm 4.15$ & $10.07 \pm 3.30$ & 2.13 & 0.039 \\
\hline$\therefore 73$ & $22.96 \pm 5.28$ & $23.55 \pm 5.35$ & $23.39 \pm 5.71$ & $23.13 \pm 4.24$ & $25.62 \pm 5.11$ & $24.93 \pm 4.01$ & $24.26 \pm 4.49$ & 2.83 & 0.007 \\
\hline 32 & $8.24 \pm 1.81$ & $8.27 \pm 1.81$ & $8.32 \pm 1.92$ & $8.63 \pm 2.01$ & $8.26 \pm 1.96$ & $8.07 \pm 2.56$ & $7.81 \pm 1.92$ & 1.10 & 0.360 \\
\hline$i .42$ & $41.83 \pm 9.09$ & $42.57 \pm 8.99$ & $42.10 \pm 10.11$ & $43.60 \pm 9.19$ & $44.75 \pm 8.59$ & $43.85 \pm 8.55$ & $42.15 \pm 7.73$ & 2.18 & 0.034 \\
\hline .35 & $33.09 \pm 8.01$ & $33.99 \pm 8.50$ & $35.27 \pm 7.73$ & $35.94 \pm 7.41$ & $36.29 \pm 9.55$ & $34.85 \pm 7.12$ & $34.89 \pm 7.27$ & 1.67 & 0.115 \\
\hline$\therefore 89$ & $22.33 \pm 4.87$ & $23.18 \pm 5.11$ & $23.95 \pm 4.46$ & $24.28 \pm 4.28$ & $24.00 \pm 5.93$ & $23.63 \pm 4.88$ & $24.63 \pm 4.57$ & 1.71 & 0.104 \\
\hline .79 & $9.76 \pm 2.70$ & $10.34 \pm 2.58$ & $10.00 \pm 2.45$ & $10.84 \pm 2.29$ & $10.90 \pm 3.12$ & $10.67 \pm 2.83$ & $10.67 \pm 2.69$ & 1.68 & 0.111 \\
\hline 5.16 & $65.18 \pm 14.77$ & $67.51 \pm 15.33$ & $69.22 \pm 13.35$ & $71.06 \pm 13.07$ & $71.19 \pm 17.78$ & $69.15 \pm 13.68$ & $70.19 \pm 13.28$ & 1.71 & 0.105 \\
\hline
\end{tabular}

\section{Supplementary Files}

This is a list of supplementary files associated with this preprint. Click to download.

- checklist1.doc 